This is the author's copy of the publication as archived with the DLR's electronic library at http://elib.dlr.de. Please consult the original publication for citation.

\title{
Dynamic Projection of Human Motion for Safe and Efficient Human-Robot Collaboration
}

Meng, Xuming; Weitschat, Roman

\section{Copyright Notice}

(C) 2021 IEEE. Personal use of this material is permitted. Permission from IEEE must be obtained for all other uses, in any current or future media, including reprinting/republishing this material for advertising or promotional purposes, creating new collective works, for resale or redistribution to servers or lists, or reuse of any copyrighted component of this work in other works.

\section{Citation Notice}

OINPROCEEDINGS $\{9561726$

author $=\{$ Meng, Xuming and Weitschat, Roman $\}$,

booktitle $=\{2021$ IFEE International Conference on Robotics and Automation (ICRA) $\}$,

title $=\{$ Dynamic Projection of Human Motion for Safe and Efficient Human-Robot Collaboration $\}$

year $=\{2021\}$

volume $=\{\}$,
number $=\{\}$

number $=\{\}$,

doi $=\{10.1109 /$ ICRA48506.2021.9561726 $\}$

\} 


\title{
Dynamic Projection of Human Motion for Safe and Efficient Human-Robot Collaboration
}

\author{
Xuming Meng and Roman Weitschat
}

\begin{abstract}
In the modern manufacturing process, novel technologies enable the collaboration between humans and robots, which increases productivity while keeping flexibility. However, these technologies also lead to new challenges, e.g., maximization of Human-Robot Collaboration (HRC) performance while ensuring safety for the human being in fenceless robot applications. In this paper, an approach of the dynamic human motion projection is proposed for typical assembly tasks. The human upper body is simplified as a five-degree-of-freedom (5DOF) rigid-body model. A control-oriented projection model is proposed, and its parameters are estimated from the test data of human capability. Combined with a human-state estimator and a collision estimator, the "worst-case" collision motion is projected in the HRC scenario. The dynamic projection method is feasible online. Finally, the estimated collision time is adopted to increase the robot's speed limit, which validates the improvement of HRC's efficiency.
\end{abstract}

\section{INTRODUCTION}

Safety is a major challenge in Human-Robot-Collaboration (HRC) in the future manufacturing and cyber-physical systems [1]. The separation between humans and robots by a protective fence is the ubiquitous safe working scenario in production. In order to achieve high productivity of a HRC system and to ensure safe operation, there is a wide range of technologies and strategies that can be used with technical or organizational protective measures. In 2011, several safety standards of HRC i.e. ISO 10218 I and II [2] were drawn up. The first collaborative robot, the lightweight robot with the collision detection function, was developed in the Institute of Robotics and Mechatronics (RM) at German Aerospace Center (DLR). Later, the large robot manufacturers, together with the technical committee, developed the technical specification ISO/TS 15066 [3], especially for collaborative robots like UR10 (Universal Robots), YuMI (ABB), IIWA (KUKA), etc. In ISO/TS 15066, the quasi-static and transient contacts are considered for the typical HRC scenes, which prescribes the maximal permissible contact force and pressure at each body region. The ${ }^{R} \dot{x}_{I S O}{ }^{1}$ representing the maximal speed limit of the robot can be measured. The threshold value is not directly defined in the ISO standard but measured by collision experiments considering the peak collision force and pressure. Thus, the corresponding maximal velocity of the robot is respect to $\left\|{ }^{R} \dot{\boldsymbol{x}}_{\max }(t)\right\|={ }^{R} \dot{x}_{I S O}$. The resulting velocity limit is depending on the mass and geometry of

\footnotetext{
The authors are with the Institute of Robotics and Mechatronics Center (RMC), German Aerospace Center (DLR), Müncher Str. 20, 82234 Wessling, Germany (E-mail: \{Xuming.Meng, Roman. Weitschat\}@dlr.de)

${ }^{1}$ The left index ${ }^{R}$ (or ${ }^{H}$ ) represents the value or vector regarding the robot (or the human).
}

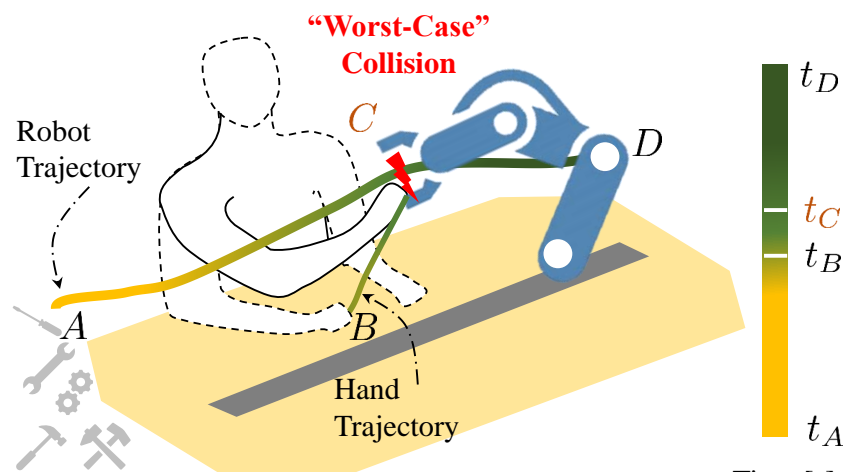

Time $[\mathrm{s}]$

Fig. 1. Schematic view of a "worst-case" collision scenario in HRC. The robot is currently inside the reachable space of the human and the human is assumed moving deliberately towards the robot with a maximal power in any position at any time.

robots. However, in some cases, such as out of the human's reachable range, the collision can never happen. The precondition of collision cases if the robot is following one certain trajectory must be: the trajectory of the robot intersects the reachable space of the human, and the human motion must be restricted to the intrinsic bio-dynamics. In addition, the collision may happen if the human deliberately moves towards the robot, cf. Fig. 1. This motion can be conscious or unconscious, for instance, a reflex movement. Thus, the robot velocity must be decelerated to the ${ }^{R} \dot{x}_{I S O}$ with the maximal deceleration before the collision happens so that the safety requirements are satisfied. In previous work [4], the aforementioned maximal allowed robot velocity is extended into:

$\left\|{ }^{R} \dot{\boldsymbol{x}}_{\max }(t)\right\|={ }^{R} \dot{x}_{I S O}+\tilde{t}_{\text {coll }}\left(t,{ }^{H} \boldsymbol{x}(t),{ }^{H, R} \boldsymbol{x}(t)\right) \cdot\left\|{ }^{R} \ddot{\boldsymbol{x}}_{\max }\right\|$,

where $\tilde{t}_{\text {coll }}$ is the estimated collision time from the current hand position ${ }^{H} \boldsymbol{x}(t)$ to the estimated collision point ${ }^{H, R} \boldsymbol{x}\left(t+\tilde{t}_{\text {coll }}\right)$ and ${ }^{R} \ddot{\boldsymbol{x}}_{\text {max }}$ is the maximal acceleration or deceleration of the robot.

In this paper, the aim is to project the human fastreaching or explosive motion ("worst-case" scenario) based on the human dynamics so that the projected motion and the collision time can maximize the efficiency of HRC, while the safety in collaborative applications is always guaranteed. The main contributions of this paper are listed as follows:

1) A five-degree-of-freedom (5-DOF) dynamics model of the human upper body is developed.

2) The features of human fast-reaching motion are sum- 
marized from the experiments, and a novel human motion projection method is proposed by utilizing the collected experimental data.

3) The projection model is online feasible and validated for the improvement of the efficiency of HRC.

\section{RELATED WORK}

In general, two mainstream methods of forecasting the fast-reaching motion in a high-dynamical scenario. The first category is with the help of a well-learned statistical model, whose input is motion-captured data, to predict successive human motion, e.g., Hidden Markov model [5], Dynamic Movement Primitives [6]. The data-driven methods are adequate for forecasting of relatively gentle or periodic motions. One concern is that purely kinematic models or models with implicit dynamics-related parameters may lead to an inaccurate or even unrealistic prediction.

The second category is by establishing a musculoskeletal model to predict the human motion. Understanding the Central-Nervous-System (CNS) and modeling the high nonlinearity in dynamics of the human body are challenging, since the human dynamics is mostly stochastic, personspecific and task-specific. In [7], the arm movement is stated as a motor control problem. The authors in [8] reveal that the human neuron-system employs specific muscle synergies during a natural fast-reaching task. In [9], authors adopt a reduced neuro-musculo-skeletal model or with a kinematic latent-space controller aiming to generate a humanlike motion to the desired position. One significant drawback of a musculoskeletal model is a drastic increase of the computational complexity. Pure skeletal model can also work well, since several researchers [10][11] have found that CNS may follow an internal optimal-control principle during planning motions. There are two categories of cost functions, kinematic and dynamic types, are partially proven to be effective by reconstructing the motion from motioncaptured data. Kinematic types include minimum hand jerk, joint acceleration, joint angle jerk [12][13]. Dynamic types include minimum torque, torque change, energy and geodesic [14][15][16]. Nevertheless, the previous research is based on only a specific cost which is with respect to a certain specific motion behavior. The authors in [17] reveal that human motion follows an optimal trajectory, which is a solution of a typical optimal control problem with a weighted combination of cost functions. Very few research concern about the difference of gentle and swift movements. Normally, the fast-reaching or explosive motion is mostly influenced by dynamic parameters such as body's inertia and the current posture [17].

\section{DyNAMiCs OF Human UPPER Body}

In the previous work [4], the torso can be assumed to be fixed. Nevertheless, e.g., suddenly taking the tool from the shelf or unconscious reflex-like motions, extreme motions of the human arm are accompanied by the torso's movement, which cannot be omitted. One DOF at the caudal vertebra (at around the height of the stomach) is added to retain the

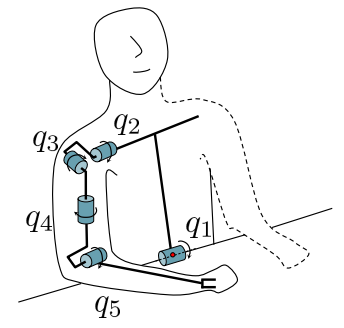

(a)

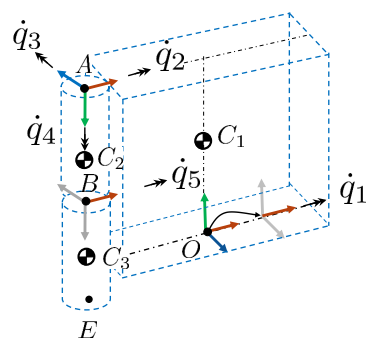

(b)
Fig. 2. Kinematic model of the 5-DOF human upper body. (a) The righthanded user is assumed to be sitting (with a fixed base) in front of DLROctagon-Workbench while collaborating with a Light-Weight-Robot (LWR), not depicted here. (b) Simplified rigid-body model in the zero-position. Leftarm, hand, and head are omitted. The letters $O, A, B, E$ are centers of the origin, the shoulder joint, the elbow joint, and TCP, respectively. The centers of mass of each body part $C_{1}, C_{2}, C_{3}$ are assumed to be lying on the center of the cube or the cylinder. The TCP $E$ is assumed locating at the center of the forearm end. The anatomical parameters for an average male's upper body can be found in [18].

moderate computation expense, considering the torso motion. Fig. 2(a) depicts the choice of DOFs. The joint space is $\boldsymbol{q} \in \mathbb{R}^{5}$. The hand position $\boldsymbol{x}^{h} \in \mathbb{R}^{3}$ is approximated as the position of Tool-Center-Point (TCP), defined as the task space or Cartesian space. The orientation of the hand is not of interest in the context of this work. The full kinematics of TCP including the position ${ }_{0} \boldsymbol{r}_{t c p}{ }^{2}$, velocity ${ }_{0} \boldsymbol{v}_{t c p}$ and Jacobian Matrix $\boldsymbol{J}_{w}=\frac{\partial_{0} \boldsymbol{v}_{t c p}}{\partial \dot{\boldsymbol{q}}}$. With the movement range of each joint given in [19], the reachable space (the light blue region) of this fixed-based model is depicted in Fig. 3. With the help of Lagrangian mechanics, the dynamics of the

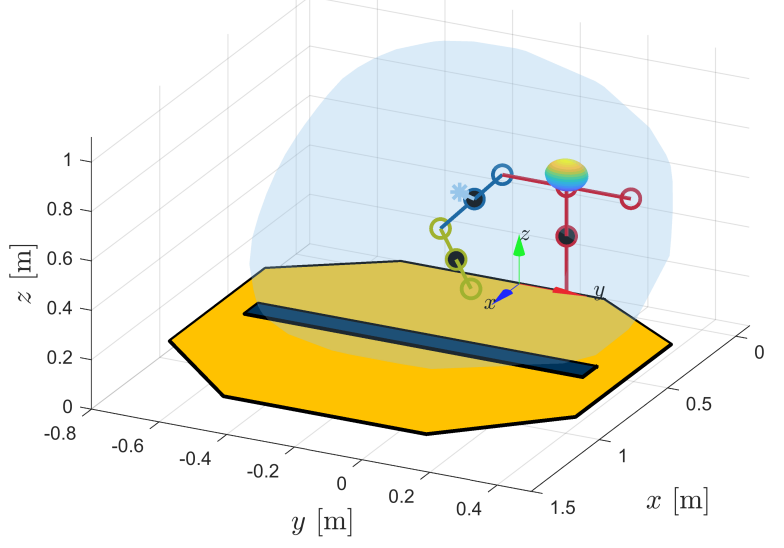

Fig. 3. Reachable workspace $\mathcal{S}$ of the fixed-base model. The human model is simplified as an equivalent skeleton-model. The solid black dot stands for the center of mass of each body part (red: torso; blue: upper arm; green: forearm). The symbol $*$ has no direct kinematic meaning; it is added to represent the actual configuration of $q_{4}$ visually. The octagon-workbench is depicted in yellow, including the linear axis (middle in black) of a LWR, not depicted here.

human model can be written as

$$
M(q) \ddot{q}+h(q, \dot{q})+g(q)=\tau+\tau^{e x t},
$$

\footnotetext{
${ }^{2}$ The left-down index 0 describes the vector measured in the Coordinate
} 0 , i.e., the world frame. 
which includes symmetric and positive definite inertia matrix $M(q) \in \mathbb{R}^{5 \times 5}$, The term denoting Coriolis and centrifugal force $h(q, \dot{q}) \in \mathbb{R}^{5}$, gravity $g(q) \in \mathbb{R}^{5}$, control input $\tau \in$ $\mathbb{R}^{5}$ control input and external forces $\tau^{e x t} \in \mathbb{R}^{5}$. In this work, an adult man with the average weight of $75 \mathrm{~kg}$ is assumed. The average dimension of each body part can be found in [18]. Since the dimension of the task space is smaller than the dimension of the joint space, the inverse kinematics [20][21] for the redundant human model is modified to

$$
\dot{\boldsymbol{q}}=\boldsymbol{J}_{W}^{\dagger} \dot{\boldsymbol{x}}_{d e s}-\alpha \boldsymbol{N} \boldsymbol{W}^{-1}\left(\frac{\partial H}{\partial \boldsymbol{q}}\right)^{T},
$$

where the weighted damped pseudo-inverse Jacobian matrix is formulated as $\boldsymbol{J}_{W}^{\dagger}=\boldsymbol{W}^{-1} \boldsymbol{J}_{w}^{T}\left(\boldsymbol{J}_{w} \boldsymbol{W}^{-1} \boldsymbol{J}_{w}^{T}+\lambda^{2} \boldsymbol{I}\right)^{-1}$. The variable $\boldsymbol{W} \in \mathbb{R}^{5 \times 5}$ is a weighting matrix. The constant $\lambda \in \mathbb{R}$ is a damping factor for avoidance of illconditioned configuration of the Jacobian matrix [21][22]. The constant $\alpha \in \mathbb{R}$ is a scalar, defining the step size for the gradient of the cost function. The modified null space projection matrix $\boldsymbol{N}=\boldsymbol{I}-\boldsymbol{J}_{W}^{\dagger} \boldsymbol{J}_{W}$. The scalar $H$ is a user-defined cost function considering task constraints e.g. to keep the human posture away from the uncomfortable or physiological impossible interval, written as $H(\boldsymbol{q})=$ $\frac{1}{2} \sum_{i=1}^{5}\left(\frac{q_{i}-q_{i, \text { comf }}}{q_{i, \text { max }}-q_{i, \text { min }}}\right)^{2}$ where the constant $q_{i, \text { comf }}=0$ is assumed as the comfortable position for the human. This solution will be implemented in inverse kinematics in the projection model in Sec. IV-C.

\section{Projection of Human Motion}

This section describes the imitation process of human motions, which can be simulated by the projection of trajectories and collision times in the "worst-case" scenario of HRC. A schematic presentation of the human motion projection and the internal connection is depicted in Fig. 4.

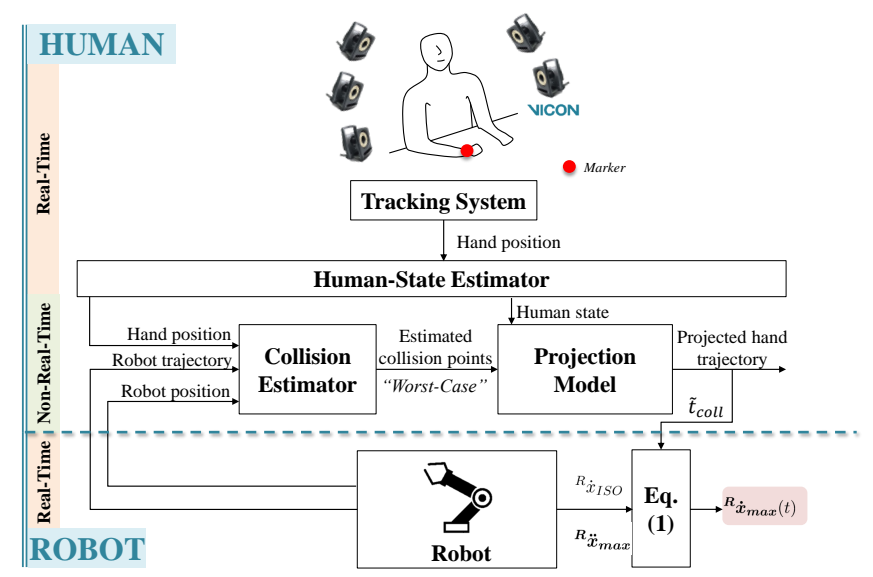

Fig. 4. Schematic structure of human motion projection. The tracking system delivers the real-time position of the human hand. The humanstate estimator is used to estimate joint positions and velocities as initial information for the projection model. The collision point estimator predicts the possible collision points for the projection model. With the final results of the projected collision time $\tilde{t}_{\text {coll }}$, the improved upper limit of the robot velocity can be calculated.

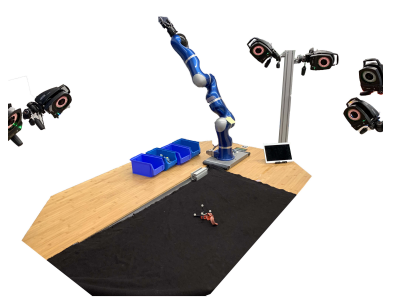

(a)

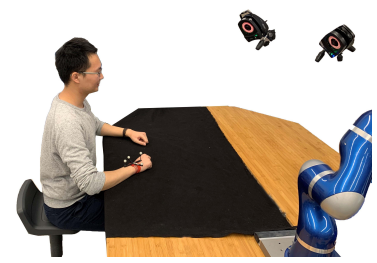

(b)
Fig. 5. Experiment setup. The experiment platform is an octagon table of HRC in the lab of "Factory of the Future" at DLR. The LWR3 with a linear conveyor band is the middle. A marker-based system VICON captures the motion. (a) VICON camera configuration and the experiment platform: LWR3 and the octagon table. (b) The subject is sitting in front of the octagon workbench. The marker is fixed at the human wrist, whose position approximates the TCP of the human.

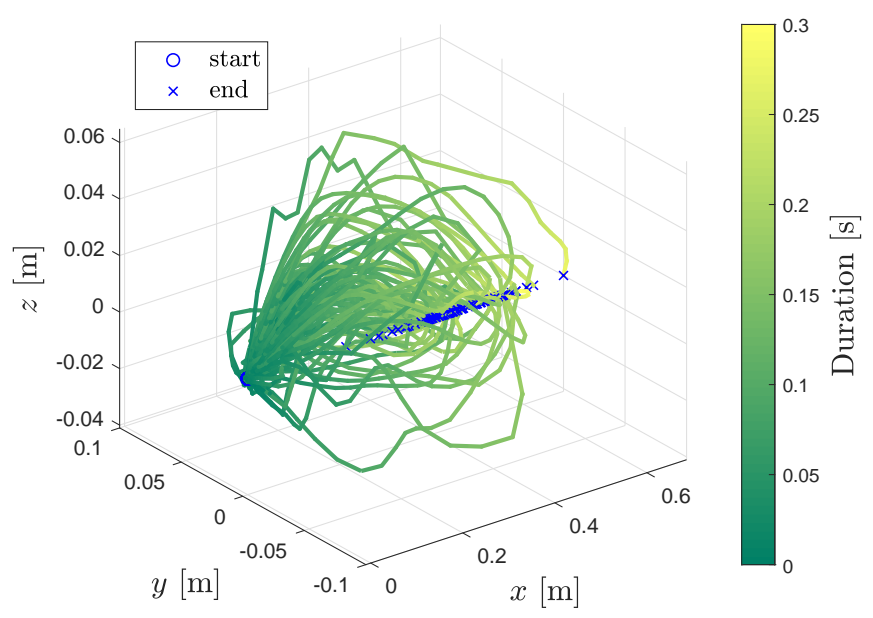

Fig. 6. Collection of 84 valid hand trajectories transformed in a local CoSys. Local transformation: Connect the start- and endpoint of the trajectory as an auxiliary line. Transform all these lines together with trajectories aligned with a certain direction (e.g., in $x$-direction). Move all start points to the same point (e.g., the origin).

\section{A. Research of Human Capability}

The following experiment is aimed to discover the underlying behavior of hand trajectories in both spatial and time domains. The experiment setup is depicted in Fig. 5. Five male subjects (right-hand users) are asked to move freely as fast as they can inside the visual field. More than 150 trajectories are recorded during the experiment. In the end, 84 valid trajectories were selected. To make those trajectories with different directions and lengths comparable, a coordination transformation is employed, cf. Fig. 6. By analyzing the covariance features of trajectories, there is no clear spatial path pattern found for the human fast-reaching motion. The next task is to find out velocity features of all trajectories. The hand speed curves $\left\|\dot{\boldsymbol{x}}^{h}(t)\right\|$ are aligned with their peaks at $\Delta t=0$, which makes different velocity curves comparable. The color of each curve characterizes quantitatively whether the subject moves upward or downward. The angle $\alpha_{z}$ between the motion direction and the plane $x y$ reflects this process. The mathematical formulation is written in $\alpha_{z}=\frac{\pi}{2}-\arccos \frac{\left(\boldsymbol{x}_{\text {end }}-\boldsymbol{x}_{\text {start }}\right) \cdot \boldsymbol{n}_{x y}}{\left\|\boldsymbol{x}_{\text {end }}-\boldsymbol{x}_{\text {start }}\right\|} \in\left[-\frac{\pi}{2}, \frac{\pi}{2}\right]$, where the 


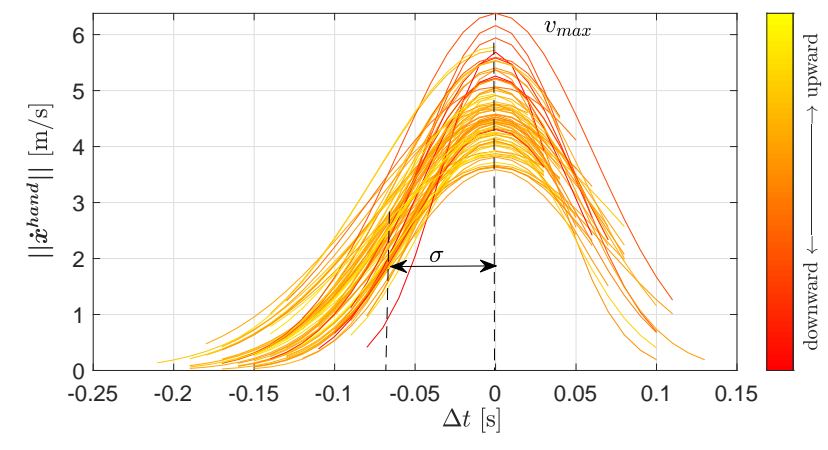

Fig. 7. The aligned Gaussian fitting of 84 hand speed curves. The $\mathrm{x}$-Axis $\Delta t$ denotes the relative time to the peak. The data points with $\Delta t<0$, representing the acceleration part of the single motion, are utilized for curve fitting. The color scaling represents the angle between the motion direction and the normal vector of plane $x y$, representing how upward or downward the hand motion is. For the "worst-case" consideration, the criteria for those valid trajectories are: 1) The average hand speed should be larger than 2.0 $\mathrm{m} / \mathrm{s}$. 2) The peak velocity should be larger than $3.5 \mathrm{~m} / \mathrm{s}$. The variable $\sigma>0$ characterizes the peak's width, assumed as the equivalent arising time.

vector $\boldsymbol{n}_{x y}=\left[\begin{array}{lll}0 & 0 & 1\end{array}\right]^{T}$ is a normal vector of the plane $x y$. The previous study [23] showed that the hand speed follows a bell-shaped profile if the hand moves along a straight line, but with a relatively slow speed (ca. $0.25 \sim 0.35 \mathrm{~m} / \mathrm{s}$ ). In this work, the rising part of the velocity curve attracts more interest, reflecting the maximal acceleration of the human hand. The results are shown in Fig. 7. The curves can be approximated by a Gaussian function, formulated as

$$
\left\|\dot{\boldsymbol{x}}^{h}\right\|=v_{\max } e^{-\frac{\Delta t^{2}}{\sigma^{2}}}
$$

where the parameter $v_{\max }>0$ represents the maximal hand velocity. Besides, by collecting all $v_{\max }$ and $\sigma$ for each curve, the both variables have linear relationships w.r.t. $\alpha_{z}$, summarized as

$$
\begin{aligned}
v_{\max }\left(\alpha_{z}\right) & =a_{1} \alpha_{z}+b_{1}, \\
\sigma\left(\alpha_{z}\right) & =a_{2} \alpha_{z}+b_{2},
\end{aligned}
$$

where the coefficients $a_{1}=-0.7833, b_{1}=5.0386, a_{2}=$ $0.027 b_{2}=0.0806$ are from the collected data. It is reasonable that for those downwards motion $\left(\alpha_{z} \downarrow\right)$, the human moves faster $\left(v_{\max } \uparrow \sigma \downarrow\right)$ due to the gravity. This relationship can also be affected by the subjects' intention, habits, and psychological factors, i.e., avoiding a collision with the workbench's surface during the experiment. Nevertheless, for the sake of simplicity, this average "worst-case" model is still implemented for the later use.

\section{B. Human-State Estimator: Compliance Control}

The human state estimator delivers estimated joint positions $\hat{\boldsymbol{q}}_{0}$ and joint velocities $\dot{\hat{\boldsymbol{q}}}_{0}$, used as the initialization of the simulation of the projection model. However, since VICON can only deliver the Cartesian hand position $\boldsymbol{x}^{h}$, estimation of joint velocities will be challenging. Employment of the closed-loop inverse kinematics at the velocity level to attain the estimated joint positions and velocities will fail due to the noisy input (position signal). Hence, inspired by the classic compliance control of kinematic redundant manipulator [24], Cartesian hand position $\boldsymbol{x}^{h}$ is regarded as the desired position. The control input $\tau$ of the system dynamics (2) can be implemented with a feedback law of the form in the following

$$
\boldsymbol{\tau}=\boldsymbol{g}(\boldsymbol{q})+\boldsymbol{J}(\boldsymbol{q})^{T} \boldsymbol{\tau}_{c}+\mathcal{N}(\boldsymbol{q}) \tau_{n},
$$

where the Cartesian compliance $\boldsymbol{\tau}_{c}=-\boldsymbol{K}\left(\boldsymbol{x}-\boldsymbol{x}^{h}\right)-\boldsymbol{D} \dot{\boldsymbol{x}}$ is specified by a symmetric and positive definite Cartesian stiffness matrix $\boldsymbol{K} \in \mathbb{R}^{m \times m}$ and a symmetric positive definite damping matrix $\boldsymbol{D} \in \mathbb{R}^{m \times m}$ at Cartesian virtual equilibrium position $\boldsymbol{x}_{h}$. The gravity term is denoted as $\boldsymbol{g}(\boldsymbol{q})$. The null space dynamics is $\boldsymbol{\tau}_{n}=-\left(\frac{\partial V_{q}(\boldsymbol{q})}{\partial \boldsymbol{q}}\right)^{T}-\boldsymbol{D}_{n} \dot{\boldsymbol{q}}$ and $V_{q, i}(\boldsymbol{q})=\sum_{1}^{n} \frac{1}{2} K_{n 0, i i}\left(q_{i}-q_{i, c o m f}\right)^{2}$ in which the null space stiffness $\boldsymbol{K}_{n 0} \in \mathbb{R}^{n \times n}$ and damping matrix $\boldsymbol{D}_{n} \in \mathbb{R}^{n \times n}$ are diagonal and positive definite. The dynamic consistent null space projection [25] is formulated as $\mathcal{N}(\boldsymbol{q})=\boldsymbol{I}-\boldsymbol{J}^{T}\left(\boldsymbol{J}^{+M}\right)^{T}$, where $\boldsymbol{I}$ is an identity matrix and the generalized weighted inverse $J^{+M}$ the defined by $\boldsymbol{J}^{+M}=\boldsymbol{M}^{-1} \boldsymbol{J}^{T}\left(\boldsymbol{J} \boldsymbol{M}^{-1} \boldsymbol{J}^{T}\right)^{-1}$ where the weighted matrix $\boldsymbol{W}$ is with the inertia matrix $M$.

\section{Projection Model}

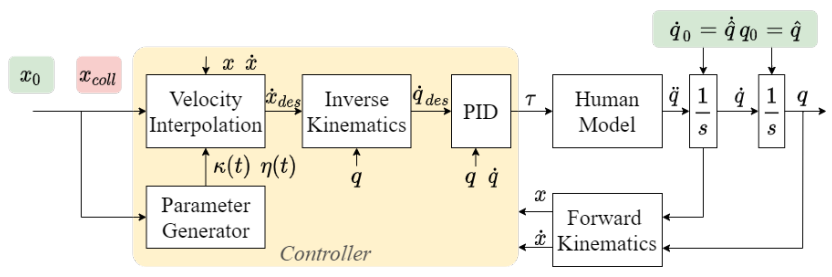

Fig. 8. Projection model: prediction of the human hand trajectory and the corresponding time span, i.e., the collision time $\tilde{t}_{\text {coll }}$ from the initial point to the estimated collision point. The signals with the green shading are online estimated from the human-state estimator, cf. Section IV-B, and the signal $\boldsymbol{x}_{\text {coll }}$ with the red shading is the result of the collision estimator, which will be illustrated in Section IV-D. Considering of a robust dynamic behavior, the simulation terminates if $\left\|\boldsymbol{x}-\boldsymbol{x}_{\text {coll }}\right\| \leq \epsilon, \epsilon=1 \sim 3 \mathrm{~cm}$.

The goal of the projection model is to estimate $\tilde{t}_{\text {coll }}$ in (1), with the known initial human state $\boldsymbol{q}_{0}$ and $\dot{\boldsymbol{q}}_{0}$, and the estimated collision point $\boldsymbol{x}_{\text {coll }}$. When the simulated hand position $\boldsymbol{x}$ arrives in the tolerance zone of the estimated collision point $\boldsymbol{x}_{\text {coll }}$, the elapsed simulation time is regarded as the collision time $\tilde{t}_{\text {coll }}$. In the practice, the projection process terminates if the Euclidean distance $\left\|\boldsymbol{x}-\boldsymbol{x}_{\text {coll }}\right\| \leq \epsilon$, e.g. $\epsilon=1 \sim 3 \mathrm{~cm}$.

The previous work in [4] shows that a velocity interpolation (admittance-control-like) strategy would be a possible solution for an automatic generation of the corresponding trajectory, cf. Fig.8. A desired Cartesian velocity $\dot{\boldsymbol{x}}_{\text {des }}=$ $\kappa \cdot \vartheta-\eta \cdot \dot{\boldsymbol{x}}$, where the direction vector is formulated as $\boldsymbol{\vartheta}=\frac{\boldsymbol{x}_{\text {coll }}-\boldsymbol{x}}{\left\|\boldsymbol{x}_{\text {coll }}-\boldsymbol{x}\right\|}$. Parameters $\kappa$ and $\eta$ reflect the effect of the muscle force of the real arm motion of humans. Compared to the constant $\kappa$ and $\eta$ in the previous work [4], a time-varied parameter is designed in a way such that the "muscle-like" virtual attraction velocity in Cartesian space, which depends on the current states of human $\left(t, \boldsymbol{x}_{0}, \dot{\boldsymbol{x}}_{0}, \boldsymbol{x}_{\text {coll }}\right)$. In Section 
$\mathrm{IV}-\mathrm{A}$, it is discovered that the parameters $v_{\max }$ and $\sigma$ in Gaussian function characterizes the natural transient/reaction time and maximal power of human motion. Consider the desired hand speed curve in a "worst-case" scenario. i.e., after reaching the maximal velocity in a transient time, the maximal value holds. (4) is then modified into a new function $f_{v}$, formulated as

$$
f_{v}\left(\Delta t_{0}, t\right)=\left\{\begin{array}{ll}
v_{\max } e^{-\frac{\left(\Delta t_{0}+t\right)^{2}}{\sigma}} & \Delta t_{0}+t<0 \\
v_{\max } & \Delta t_{0}+t \geq 0
\end{array},\right.
$$

where $v_{\max }$ and $\sigma$ are substituted by (5) and (6), respectively. The relative time $\Delta t_{0}$ represents the current motion phase of the hand, determined by

$$
\Delta t_{0}=\left\{\begin{array}{ll}
-\sqrt{-\ln \frac{10^{-3}}{v_{\text {max }}}} \cdot \sigma & \left\|\dot{\boldsymbol{x}}_{0}\right\| \leq 10^{-3} \\
-\sqrt{-\ln \frac{\left\|\dot{\boldsymbol{x}}_{0}\right\|}{v_{\max }}} \cdot \sigma & 10^{-3}<\left\|\dot{\boldsymbol{x}}_{0}\right\|<v_{\max } \\
0 & \left\|\dot{\boldsymbol{x}}_{0}\right\| \geq v_{\max }
\end{array} .\right.
$$

It is found out that $\kappa$ has a quasi-linear relation with the static part of $f_{v}$ (when $\Delta t_{0}+t \geq 0$ ). We assume this relation $\kappa=c_{1} \cdot f_{v}\left(t, \boldsymbol{x}_{0}, \dot{\boldsymbol{x}}_{0}, \boldsymbol{x}_{\text {coll }}\right)+c_{0}$, where the parameters $c_{0}=$ 1.6211, $c_{1}=4.2571$. The damping term is formulated as $\eta=2 \xi \sqrt{\kappa}$, where the modal damping factor is given by $\xi=0.6$. Hence, the velocity behavior of the projection model has a similar shape of the velocity response of a critically damped second-order system. Since the parameters related to the null space dynamics have less impact on the velocity in Cartesian space, other parameters except for $\kappa(t)$ and $\eta(t)$ can be approximated as follows $\alpha=5, \lambda=0.1, \boldsymbol{W}=$

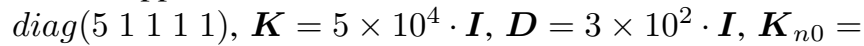
$\operatorname{diag}(55033165033165)$ and $\boldsymbol{D}_{n 0}=1.4 \cdot \sqrt{\boldsymbol{K}_{n 0}}$.

\section{Collision Estimation}

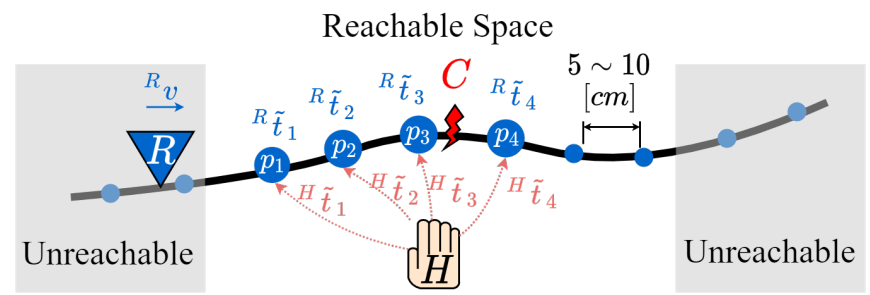

Fig. 9. Schematic diagram of the determination of the possible collision point. The symbols $R$ and $H$ stand for the current TCP of the robot and the current TCP of the human respectively. The letter $C$ denotes the final estimated collision point.

The collision point is where the condition ${ }^{R} \tilde{t}_{i}={ }^{H} \tilde{t}_{i}{ }^{3}$ fulfills. However, ${ }^{H} \tilde{t}_{i}$ can not be calculated, if the collision point is not given. Therefore, collision estimation is to "guess" an accurate collision point, while the computational load should remain low. The first step is to segment a continuous robot trajectory by adding several auxiliary points

\footnotetext{
${ }^{3}$ The time span ${ }^{H} \tilde{t}_{i}$ represents the estimated human motion time span until the $i$-th auxiliary point of the robot trajectory and the time span $R_{t_{i}}$ represents the time span for the robot moving from the current position to the $i$-th auxiliary point of its trajectory.
}

$\boldsymbol{p}^{a u x}$, in which the points inside the reachable space: $\boldsymbol{p}^{i n}=$ $\left\{\boldsymbol{p} \in \mathbb{R}^{3} \mid \boldsymbol{p}^{a u x} \subseteq \mathcal{S}\right\}$. There exists a trade-off between computational complexity and accuracy for the collision point determination. One reasonable choice of the segment length is the adult male's fist-width $\Delta x_{\text {fist }}=5 \sim 10 \mathrm{~cm}$. It is ensured that the hand collides at least with one auxiliary point, and the projected trajectory is still reasonable, see the segmentation (blue points) for a left-to-right motion in Fig. 9. The second step is to find a segment, whose two end points fulfill the conditions $R \tilde{t}_{i} \leq{ }^{H} \tilde{t}_{i}$ and $R \tilde{t}_{i+1} \geq{ }^{H} \tilde{t}_{i+1}$ at the same time. The collision point $\boldsymbol{x}_{\text {coll }}$ can be assumed at the middle point of this segment $\left(\boldsymbol{p}_{i}^{i n}+\boldsymbol{p}_{i+1}^{i n}\right) \cdot 0.5$ and the collision time $\tilde{t}_{\text {coll }}$ is approximated by the projection model with new target point $\boldsymbol{x}_{\text {coll }}$. The algorithm checks the condition for two endpoints on every segment in the finite set $\mathcal{S}$, and it terminates when the first valid segment is founded.

\section{EXPERIMENTAL VALIDATION}

In the following, the aforementioned human-state estimator and the projection model are online tested. The SIMULINK model is compiled and run in a real-time PC (Intel(R) Core(TM) i7 CPU $4.00 \mathrm{GHz}, 8 \mathrm{~GB}$ RAM).

\section{A. Online Simulation of Human-State Estimator}

The subject is asked to move freely (inclusive fast motions) in the workspace. The estimated joint position $\hat{\boldsymbol{q}}, \dot{\hat{q}}$ and Cartesian hand velocity $\dot{\boldsymbol{x}}$ are logged. The corresponding human state variables are depicted in Fig. 10. A first-order low-pass filter with time constant $10 \mathrm{~ms}$ is implemented at the input of position signal in case of the deterioration of the model's dynamic response. Despite the time delay, the filtered signal has a relatively good quality and the simulated (estimated) hand position can rapidly follow the desired position due to the large stiffness in Cartesian space. The corresponding joint positions and velocities are depicted in the last two rows of Fig. 10.

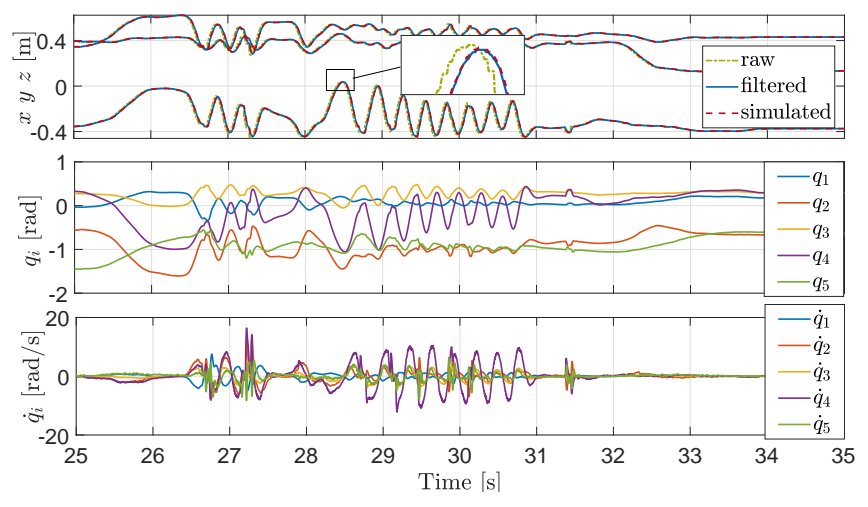

Fig. 10. Online simulation of the human-state estimator. The first row: raw, filtered, and simulated Cartesian position signals. The second and the third row: the estimated joint positions and velocities.

\section{B. Test of Projection Model}

In (1), the maximal allowed ISO velocity for the robot at the collision point is assumed as a constant ${ }^{R} \dot{x}_{I S O}=$ 


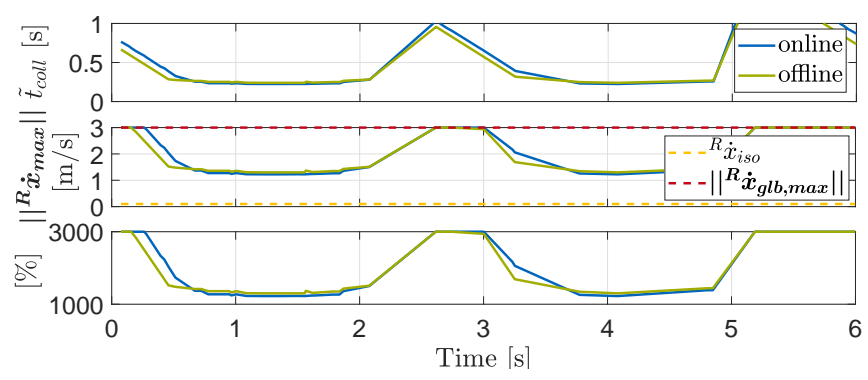

Fig. 11. The comparison of the online and the offline "resting-hand" test. Top: The estimated collision time. Middle: The improved allowed maximal robot velocity. The global maximal velocity of the robot is set as $3 \mathrm{~m} / \mathrm{s}$ (red dash line), and the ISO velocity is set as $0.1 \mathrm{~m} / \mathrm{s}$ (yellow dash line). Bottom: The improved efficiency, defined as $\frac{\left\|{ }^{R} \dot{\boldsymbol{x}}_{\max }(t)\right\|-{ }^{R} \dot{x}_{I S O}}{R_{\dot{x}_{I S O}}} \cdot 100 \%$.

$0.1 \mathrm{~m} / \mathrm{s}$, the maximal deceleration of robot is set with $\left\|^{R} \ddot{\boldsymbol{x}}\right\|_{\max }=5 \mathrm{~m} / \mathrm{s}^{2}$ and the global maximal velocity of robot is set as $\left\|{ }^{R} \dot{\boldsymbol{x}}_{g l b, \text { max }}\right\|=3 \mathrm{~m} / \mathrm{s}$. The robot is assumed to move along a straight line back and forth in the space with a trapezoidal velocity profile in Cartesian space (maximal speed: $0.6 \mathrm{~m} / \mathrm{s}$ ). The projection strategy, cf. Fig. 8 , is firstly online implemented, while the results $\tilde{t}_{\text {coll }}$ and $\left\|{ }^{R} \dot{\boldsymbol{x}}_{\text {max }}(t)\right\|$ are recorded. Meanwhile, the same projection strategy will perform offline again with recorded hand trajectories for each online simulation. We finally put the results $\tilde{t}_{c o l l}$ and $\left\|^{R} \dot{\boldsymbol{x}}_{\text {max }}(t)\right\|$ in the same figure. Two test scenarios are given in the following:

"Resting-hand" Test: The subject sits in a resting position in front of the workbench, and the hand is resting at the middle point of the workspace $\mathcal{S}$ in the $y$-axis. The comparison of final results is shown in Fig. 11. The Cartesian position error of the estimated collision is always within the $\pm 10 \mathrm{~cm}$, which meets the assumption of the online error tolerance. The large error occurs especially when the estimated collision point is close to or outside the boundary of the human reachable space, when the human is yet far away from the this point. For those estimated collision points which are far from the boundary region (between the time interval $t=0.8 \sim 1.8 \mathrm{~s}$ and $t=3.8 \sim 4.8$ s.), the errors of the collision position and time are within an acceptable tolerance $(8 \mathrm{~cm}$ and $20 \mathrm{~ms}$ ). Compared with the standard ISO-velocity $0.1 \mathrm{~m} / \mathrm{s}$, the lowest upper-limit is already larger than $1.4 \mathrm{~m} / \mathrm{s}$, cf. the bottom of Fig. 11, indicating a considerable improvement (14 times) of the HRC efficiency while maintaining the safety.

"Moving-Hand" Test: The human is asked to move in the workspace freely for 25 seconds. The results $\boldsymbol{x}_{\text {coll }}$ and $t_{\text {coll }}$ are online recorded, compared with offline results in the postprocessing, cf. Fig. 13. Similar to the results in the "restinghand" test, for those estimated collision points inside the reachable space but away from the boundary $(-0.4 \leq y \leq$ $0.0 \mathrm{~m})$, the errors are small. However, at some time points, the error can still be large since there may exist a time when the human is far from the robot. It leads directly to a longer computation time of the projection model and results in an enormous time delay in the real-time PC. This problem could
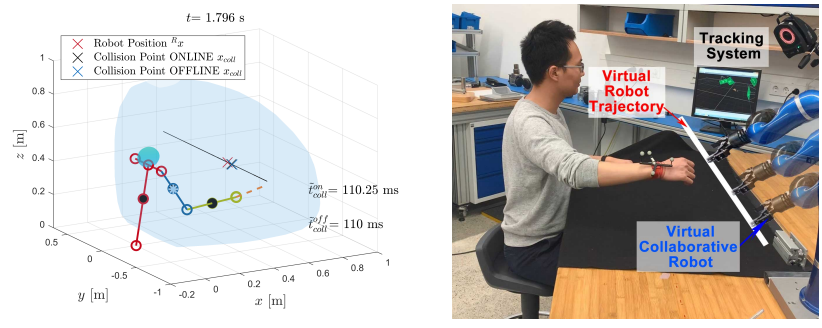

Fig. 12. Online test scenarios at certain time points for the "moving-hand" test. The tracking system captures the only Cartesian position of the right hand. The end-effector of the LWR is assumed to move along a line back and forth in the workspace. The projected hand trajectory is neglected.

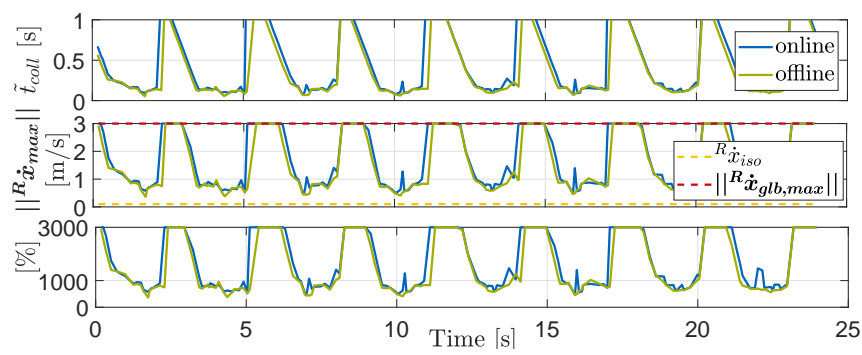

Fig. 13. The comparison of the online and the offline "moving-hand" test. Top: The estimated collision time. Middle: The improved allowed maximal robot velocity. Bottom: The improved efficiency in percentage.

be solved by enlarging the segment of the robot trajectory. Nevertheless, the efficiency is still enhanced at least 5 times, cf. the bottom of Fig. 13. In summary, the results from the online implementation are reasonable within the assumption, and HRC's efficiency can be improved in the real application.

\section{CONCLUSION}

In this paper, an approach for the human motion projection is proposed, aiming to maximize human-robot collaboration efficiency while the safety requirements are always fulfilled. It has been proven that the dynamics of the human body have a huge impact on the motion behavior. Hence, a 5DOF equivalent rigid-body model is firstly illustrated by additionally considering the movement of the torso. Later, a dynamic motion projection method for the human is introduced. The 84 valid hand trajectories from subjects are recorded and used to adjust the projection model close to a natural capable motion of the human. The human-state estimator and the projection model are online implemented. The final improved velocity limit of the robot is calculated, showing that the efficiency can be enormously improved in most cases, as long as there exists a certain distance between the human and the robot. In the future work, the improved speed limit can be used as a priori knowledge for the realtime trajectory planning of the robot, so that a more flexible and general HRC scenario can be realized.

\section{ACKNOWLEDGMENT}

I would like to express my gratitude to Prof. Alois Knoll for his suggestions to this work. 


\section{REFERENCES}

[1] S. M. M. Rahman, "Cyber-physical-social system between a humanoid robot and a virtual human through a shared platform for adaptive agent ecology," IEEE/CAA Journal of Automatica Sinica, vol. 5, no. 1, pp. 190-203, jan 2018. doi: 10.1109/jas.2017.7510760

[2] ISO, "Robots and robotic devices -safety requirements for industrial robots -part 1: Robots (iso 10218-1:2011); german version en iso 10218-1:2011," ISO, 2011.

[3] ISO, "Robots and robotic devices - collaborative robots (iso/ts 15066:2016)," ISO, 2017.

[4] R. Weitschat, J. Ehrensperger, M. Maier, and H. Aschemann, "Safe and efficient human-robot collaboration part i: Estimation of human arm motions," in 2018 IEEE International Conference on Robotics and Automation (ICRA). IEEE, may 2018. doi: 10.1109/icra.2018. 8461190

[5] H. Liu and L. Wang, "Human motion prediction for human-robot collaboration," Journal of Manufacturing Systems, vol. 44, pp. 287294, Jul. 2017. doi: 10.1016/j.jmsy.2017.04.009

[6] S. Schaal, "Dynamic movement primitives -a framework for motor control in humans and humanoid robotics," in Adaptive Motion of Animals and Machines. Springer-Verlag, 2006, pp. 261-280.

[7] F. Campos and J. Calado, "Approaches to human arm movement control-a review," Annual Reviews in Control, vol. 33, no. 1, pp. 69-77, apr 2009. doi: 10.1016/j.arcontrol.2009.03.001

[8] A. dAvella, A. Portone, L. Fernandez, and F. Lacquaniti, "Control of fast-reaching movements by muscle synergy combinations," Journal of Neuroscience, vol. 26, no. 30, pp. 7791-7810, Jul. 2006. doi: 10. 1523/jneurosci.0830-06.2006

[9] N. Ogihara and N. Yamazaki, "Generation of spontaneous reaching movement based on human anatomical constraints." Transactions of the Japan Society of Mechanical Engineers Series C, vol. 67, no. 659, pp. 2314-2320, 2001. doi: 10.1299/kikaic.67.2314

[10] N. Tsiantis, E. Balsa-Canto, and J. R. Banga, "Optimality and identification of dynamic models in systems biology: an inverse optimal control framework," Bioinformatics, vol. 34, no. 14, pp. 2433-2440, mar 2018. doi: 10.1093/bioinformatics/bty 139

[11] S. Albrecht, "Modeling and numerical solution of inverse optimal control problems for the analysis of human motions," Ph.D. dissertation, Technischen Universitat Munchen, 2013.

[12] T. Flash and N. Hogan, "The coordination of arm movements: an experimentally confirmed mathematical model." The Journal of neuroscience : the official journal of the Society for Neuroscience, vol. 5, pp. 1688-1703, Jul. 1985.

[13] Y. Wada, Y. Kaneko, E. Nakano, R. Osu, and M. Kawato, "Quantitative examinations for multi joint arm trajectory planning-using a robust calculation algorithm of the minimum commanded torque change trajectory," Neural Networks, vol. 14, no. 4-5, pp. 381-393, may 2001. doi: 10.1016/s0893-6080(01)00026-0

[14] W. L. Nelson, "Physical principles for economies of skilled movements," Biological Cybernetics, vol. 46, no. 2, pp. 135-147, feb 1983. doi: $10.1007 / \mathrm{bf} 00339982$

[15] B. Berret, C. Darlot, F. Jean, T. Pozzo, C. Papaxanthis, and J. P. Gauthier, "The inactivation principle: Mathematical solutions minimizing the absolute work and biological implications for the planning of arm movements," PLoS Computational Biology, vol. 4, no. 10, p. e1000194, oct 2008. doi: 10.1371/journal.pcbi.1000194

[16] A. Biess, D. G. Liebermann, and T. Flash, "A computational model for redundant human three-dimensional pointing movements: Integration of independent spatial and temporal motor plans simplifies movement dynamics," Journal of Neuroscience, vol. 27, no. 48, pp. 13045 13 064, nov 2007. doi: 10.1523/jneurosci.4334-06.2007

[17] O. S. Oguz, Z. Zhou, S. Glasauer, and D. Wollherr, "An inverse optimal control approach to explain human arm reaching control based on multiple internal models," Scientific Reports, vol. 8, no. 1, apr 2018. doi: 10.1038/s41598-018-23792-7

[18] R. Dumas, L. Chèze, and J.-P. Verriest, "Adjustments to McConville et al. and young et al. body segment inertial parameters," Journal of Biomechanics, vol. 40, no. 3, pp. 543-553, jan 2007. doi: 10.1016/j. jbiomech.2006.02.013

[19] T. Sawyers, "Understanding the normal shoulder range of motion what makes up your shoulder joint?" 2018. [Online]. Available: https://www.healthline.com/health/shoulder-range-of-motion

[20] B. Siciliano and O. Khatib, Eds., Springer Handbook of Robotics. Springer Berlin Heidelberg, 2008.
[21] Y. Nakamura and H. Hanafusa, "Inverse kinematic solutions with singularity robustness for robot manipulator control," Journal of Dynamic Systems, Measurement, and Control, vol. 108, no. 3, p. 163, 1986. doi: $10.1115 / 1.3143764$

[22] A. S. Deo and I. D. Walker, "Overview of damped least-squares methods for inverse kinematics of robot manipulators," Journal of Intelligent \& Robotic Systems, vol. 14, no. 1, pp. 43-68, sep 1995. doi: 10.1007/bf01254007

[23] W. Abend, E. Bizzi, and P. Morasso, "Human arm trajectory formation," Brain, vol. 105, no. 2, pp. 331-348, 1982. doi: 10.1093/brain/ 105.2.331

[24] C. Ott, A. Kugi, and Y. Nakamura, "Resolving the problem of non-integrability of nullspace velocities for compliance control of redundant manipulators by using semi-definite lyapunov functions," in 2008 IEEE International Conference on Robotics and Automation. IEEE, may 2008. doi: 10.1109/robot.2008.4543500

[25] A. Dietrich, T. Wimbock, and A. Albu-Schäffer, "Dynamic wholebody mobile manipulation with a torque controlled humanoid robot via impedance control laws," in 2011 IEEE/RSJ International Conference on Intelligent Robots and Systems. IEEE, sep 2011. doi: 10.1109/ iros.2011.6094445 